\title{
Variación temporal de especies de corales pétreos dominantes en arrecifes del Parque Nacional Jardines de la Reina, Cuba
}

\section{Temporal variation of dominant stony coral species in reefs of the Jardines de la Reina National Park, Cuba}

\author{
Leslie Hernández Fernández ${ }^{1}$
}

\begin{abstract}
RESUMEN
En el Caribe, los arrecifes coralinos han estado en constante cambio, como consecuencia de los impactos locales y globales. Se espera que tales modificaciones también ocurran en el Parque Nacional Jardines de la Reina. Por ello, este trabajo se propuso analizar la variación temporal de las poblaciones de $S$. siderea, Orbicella spp., A. agaricites y P. astreoides, como grupo dominante, entre el 2001, 2012 y 2017. Estas especies han mostrado cambios en otros arrecifes de Cuba y del Caribe. En el 2001 y 2012, se estudiaron 14 sitios, con 88 transectos cada año y en el 2017, 24 sitios y 240 transectos. En el 2001, se utilizó metodología AGRRA; en el 2012 y 2017 fue empleada una versión de esta. Se contaron 6501 colonias y se identificaron 35 especies. $S$. siderea tuvo una abundancia $(P=0.6861)$ y densidad $(P=0.0712)$ sin diferencias significativas. Orbicella spp. disminuyó en abundancia y densidad entre $2001\left(24 \%-3.2\right.$ colonias $\left.10 \mathrm{~m}^{-1}\right)$, $2012\left(14 \%\right.$ - 1.4 colonias $\left.10 \mathrm{~m}^{-1}\right)$ y $2017\left(13 \%\right.$ - 1.9 colonias $\left.10 \mathrm{~m}^{-1}\right)$. A. agaricites mantuvo su abundancia sin diferencias significativas $(P=0.2202)$ y aumentó su densidad (2001-2012: 1.4 colonias $10 \mathrm{~m}^{-1}, 2017: 2$ colonias $\left.10 \mathrm{~m}^{-1}\right)$. P. astreoides incrementó su abundancia (2001: 8\%, 2012: $10 \%$, 2017: 12\%) y densidad (2001 y 2012: 1 colonia $10 \mathrm{~m}^{-1}, 2017: 1.7$ colonias $10 \mathrm{~m}^{-1}$ ). $S$. siderea dominó en los arrecifes y Orbicella spp. tuvo una disminución que generó variación temporal con desplazamiento hacia $A$. agaricites y $P$. astreoides.
\end{abstract}

Palabras clave: arrecifes, Siderastrea, Orbicella, Agaricia, Porites.

\begin{abstract}
In the Caribbean, coral reefs have been changing constantly as a result of local and global impacts. These changes are also expected to occur in the Jardines de la Reina National Park. The work described here has been carried out to analyze the temporal variation of populations of the dominant groups $S$. siderea, Orbicella spp., A. agaricites and P. astreoides during 2001, 2012 and 2017, which have been found to have changed in other reefs of Cuba and the Caribbean. During 2001 and 201214 sites were surveyed in 88 transects every year, and during 2017 at 24 sites in

1 Centro de Bioplantas. Universidad "Máximo Gómez Báez" de Ciego de Ávila. Carretera a Morón, km 9. Ciego de Ávila.Cuba. CP: 65100. leslie@bioplantas.cu, coraleslhf@gmail.com. ORCID: http://orcid.org/0000-0002-1939-9790
\end{abstract}


240 transects. In 2001, the AGRRA methodology was used; in 2012 and 2017, a new version of this methodology was applied. Six thousand five hundred and one $(6,501)$ colonies were counted and 35 species were identified. S. siderea had an abundance $(P=0.6861)$ and density $(P=0.0712)$ which did not vary significantly between the different surveys. The abundance and density of Orbicella spp. decreased between $2001\left(24 \%\right.$ - 3.2 colonies $\left.10 \mathrm{~m}^{-1}\right), 2012(14 \%-1.4$ colonies $\left.10 \mathrm{~m}^{-1}\right)$, and $2017\left(13 \%-1.9\right.$ colonies $\left.10 \mathrm{~m}^{-1}\right)$. The abundance of $A$. agaricites also did not vary significantly between the different surveys $(P=0.2202)$, while its density increased (2001-2012: 1.4 colonies $10 \mathrm{~m}^{-1}, 2017: 2$ colonies $\left.10 \mathrm{~m}^{-1}\right)$. The abundance of $P$. astreoides increased (2001: 8\%, 2012: 10\%, 2017: 12\%) as did its density (2001- 2012: 1 colony $10 \mathrm{~m}^{-1}, 2017: 1.7$ colonies $\left.10 \mathrm{~m}^{-1}\right)$. The coral $S$. siderea prevailed in the reefs, and Orbicella spp. underwent a reduction that caused temporal variation with a displacement toward $A$. agaricites and $P$. astreoides.

Keywords: reefs, Siderastrea, Orbicella, Agaricia, Porites.

\section{INTRODUCCIÓN}

Entre los ecosistemas marinos, es conocida la importancia de los arrecifes coralinos (Gil-Agudelo et al. 2020), los cuales son altamente productivos y biodiversos, pero están amenazados por el estrés local y global (Hoegh-Guldberg et al. 2019). Las causas del estrés local contemplan variedad de actividades humanas, como las pesquerías y el desarrollo costero. En cuanto al estrés global, una de las mayores amenazas para la salud de los arrecifes coralinos es el cambio climático, que ocasiona el blanqueamiento asociado a las inusuales altas temperaturas de la superficie del mar (Obura \& Grimsdith, 2009), así como la acidificación de los océanos (Hoegh-Guldberg et al. 2017).

Los impactos locales y globales se toman como las razones de cambio de fase de un grupo de especies hacia otros, lo que resulta cada vez más común sobre los arrecifes coralinos $y$, escalonadamente, en muchos otros ecosistemas (Hughes et al. 2010). Tal vez la modificación más conocida es el reemplazo de corales por algas, pero ocurren otras cuando el sistema experimenta una fuerte bioerosión (Mumby $\&$ Steneck, 2011). Por ejemplo, el género Orbicella, considerado el más importante de los corales constructores de arrecifes en el Caribe, ha sufrido un drástico declive asociado a los efectos negativos de las actividades humanas, episodios de enfermedades y eventos de blanqueamiento (Hayes, 2019; Toth et al. 2019; Yranzo, 2020).

Específicamente, en el sur del Caribe, Orbicella spp. se ha visto en mayor riesgo por la enfermedad de la banda amarilla, lo que se asocia a las altas temperaturas de la superficie del mar (van Woesik \& Randall, 2017). En las Islas Vírgenes (EE. UU.), la especie Orbicella annularis (Ellis \& Solander, 1786) ha sido afectada por huracanes y el blanqueamiento, sin 
embargo, muestra algunos signos de recuperación (Edmunds, 2015).

Porites astreoides Lamarck, 1816, a pesar de todas las consecuencias que ha traído el estrés local y global para la comunidad coralina, se ha mantenido como una especie de éxito para el Caribe (Eagleson et al. 2021). En ello puede estar influyendo su estrategia de reproducción (Goodbody-Gringley \& Putron, 2016) y su tolerancia tanto a un intervalo amplio de temperatura como a las condiciones físicas y de calidad de las aguas (Baumann et al. 2016), por lo que es de gran plasticidad y oportunista (Jackson et al. 2014; De Bakker et al. 2016).

Según Green et al. (2008), en el Caribe, la estructura de la comunidad de los arrecifes coralinos está cambiando, sobre una escala de tiempo de diez años, pasando a ser dominada por corales "weedy" de crecimiento rápido, de pequeño tamaño, ciclo de vida corto y rápido reemplazo. Específicamente, Orbicella spp. está siendo sustituida por otras especies como P. astreoides y Agaricia spp. (Jackson et al. 2014; De Bakker et al. 2016), como reflejo de la respuesta de los arrecifes coralinos ante el estrés global (Bruno et al. 2019).

En Cuba, Alcolado et al. (2009), en un estudio realizado en el golfo de Batabanó, observaron que el predominio de $O$. annularis (como complejo de especies) -para este entonces se incluía a Montastraea cavernosa (Linnaeus, 1767)- estaba siendo desplazado por el aumento de P. astreoides y Siderastrea siderea (Ellis \& Solander, 1786) -en distintas combinaciones-. González-Díaz et al. (2018), en su investigación sobre el estado de los arrecifes coralinos en Cuba, reportaron entre las especies más abundantes a $P$. astreoides y a $S$. siderea, y con una baja abundancia a Orbicella spp., lo que evidenció una degradación ecológica similar a la del Caribe. Comparados con los años 80, según Caballero et al. (2019), los arrecifes cubanos mantienen la misma riqueza de especies, pero la dominancia ha cambiado de especies como Acropora palmata (Lamarck, 1816) у $O$. annularis hacia otras oportunistas como $S$. siderea y Agaricia agaricites (Linnaeus, 1758).

En el Parque Nacional Jardines de la Reina (PNJR) se han registrado como especies dominantes, para los escarpes pocos profundos, $S$. siderea, A. agaricites y $P$. astreoides (Hernández-Fernández et al. 2011; Ferrer-Rodríguez et al. 2016; Hernández-Fernández et al. 2019a; Hernández-Fernández et al. 2019b) y, como moderadamente común, Orbicella spp. (González-Díaz et al. 2018; Hernández-Fernández et al. 2019b), hábitat que se caracteriza por presentar una estructura en la comunidad coralina, con alta variabilidad a pequeña escala- $10^{1} \mathrm{~m}$ - (Hernández-Fernández et al. 2019b). Todos estos estudios han tenido un enfoque general en la comunidad coralina, sin 
analizar directamente los indicadores ecológicos por especies. Cabría preguntarse si en una de las áreas marinas protegidas (AMP) más importantes de Cuba (Perera-Valderrama et al. 2018) también existe una variación espacial de especies dominantes.

Las transformaciones que han ocurrido en las poblaciones de S. siderea, Orbicella spp., A. agaricites y $P$. astreoides, en otros arrecifes de Cuba (Alcolado et al. 2009) y en el Caribe (Jackson et al. 2014; De Bakker et al. 2016), igualmente se espera que sucedan en el Parque Nacional Jardines de la Reina (PNJR), donde ello no se ha constatado con anterioridad, así que este trabajo se propuso analizar la variación temporal de ese grupo de especies dominantes, desde el 2001 hasta el 2017. Esta información sería de gran utilidad para los tomadores de decisiones del parque, pues les permitiría tomar las medidas pertinentes ante un posible deterioro del arrecife.

\section{MATERIALES Y MÉTODOS}

El Parque Nacional Jardines de la Reina (PNJR) se encuentra en la República de Cuba, específicamente, en el archipiélago de los Jardines de la Reina, al sur de las provincias centrales de Sancti Spíritus, Ciego de Ávila y Camagüey. Lo conforman alrededor de 661 cayos y abarca un total de 217 ha, aproximadamente, de las cuales 200 ha son marinas (Perera-Valderrama et al. 2018). Precisamente por sus valores ecológicos y de conservación, a toda la región del archipiélago le fue otorgada la categoría de parque nacional, dado el acuerdo 6803/2010 del Comité Ejecutivo del Consejo de Ministros de Cuba.

Los arrecifes estudiados se ubican al sur de los cayos y corresponden con el hábitat de escarpes poco profundos (8-15 $\mathrm{m}$ de profundidad) (Fig. 1), donde los fondos son irregulares, duros, arenosos y cuyos hábitats son considerados homogéneos (Pina-Amargós et al. 2008). Estos arrecifes se caracterizan por presentar crestas someras con predominio de Millepora complanata (Lamarck, 1816), A. palmata y $P$. astreoides, así como escarpes poco profundos donde predominan las especies de corales $S$. siderea, A. agaricites y los octocorales Pseudopterogorgia americana Gmelin 1791 y Eunicea flexuosa Lamouroux 1821; aquí se desarrolla buceo recreativo (Hernández-Fernández et al. 2016). Muy diferentes resultan los fondos del norte de los cayos, caracterizados por ser predominantemente fangosos, con cabezos de corales y crestas aisladas, donde en gran parte del año prevalecen sedimentos suspendidos $\mathrm{y}$ poca visibilidad; P. astreoides y Porites furcata Lamarck, 1816 son las especies preponderantes (Ferrer-Rodríguez et al. 2016). 


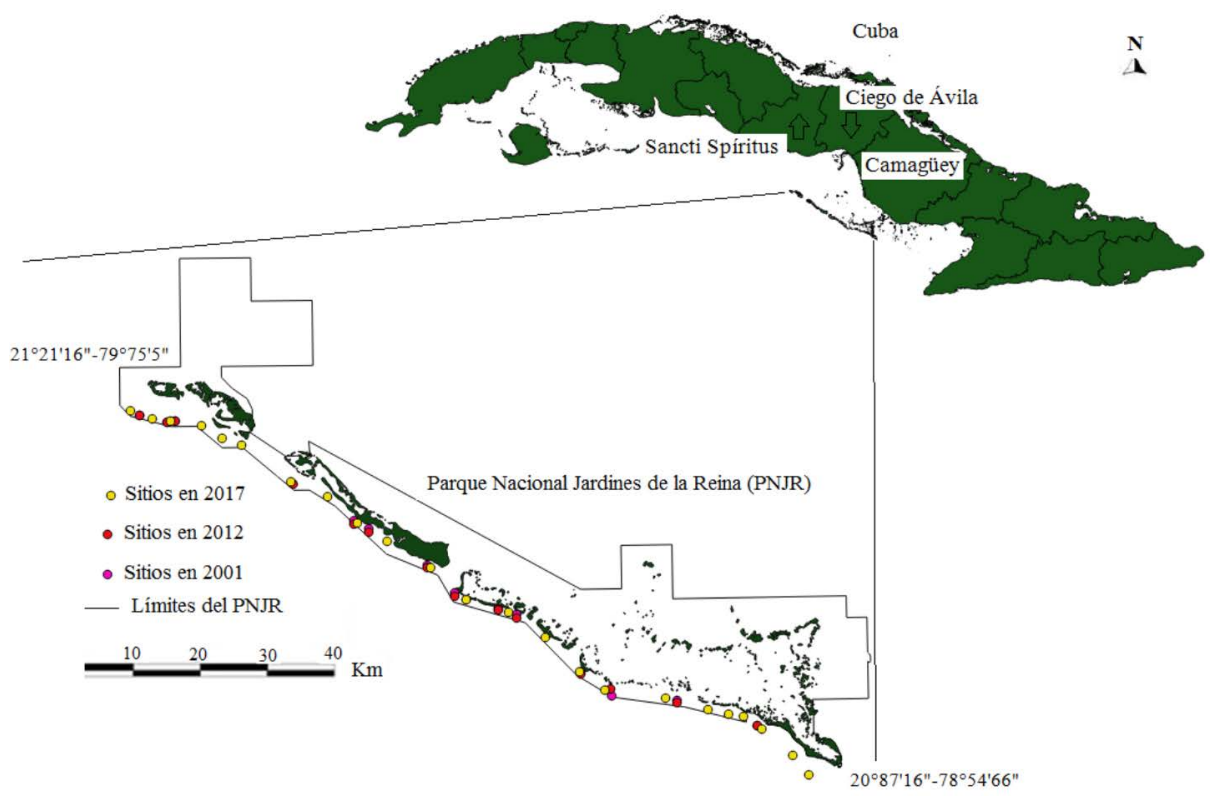

Fig. 1. Sitios de muestreos, estudiados en el 2001, 2012 y 2017, en el hábitat de escarpes poco profundos del Parque Nacional Jardines de la Reina

Fig. 1. Sampling sites studied in 2001, 2012 and 2017 in the reef slope habitat of the Jardines de la Reina National Park

Para examinar la variación temporal de las poblaciones del grupo de especies dominantes $S$. siderea, Orbicella ssp. A. agaricites y $P$. astreoides en el PNJR, se realizó un análisis, fundamentalmente, de los indicadores ecológicos de abundancia relativa (\%) y densidad lineal promedio de colonias (colonias 10 $\mathrm{m}^{-1}$ ) de estas especies, en los años 2001, 2012 y 2017. Con tal fin, se usó parte de los datos obtenidos en la expedición conjunta CUBAGRRA II, realizada en agosto de 2001, en la cual se aplicó la metodología AGRRA (2000). También se empleó parte de la información obtenida en expediciones realizadas en abril de 2012 y septiembre-octubre de 2017, en las que se puso en práctica la metodología propuesta por Caballero et al. (2013). Ambas metodologías emplean el método del transecto lineal de $10 \mathrm{~m}$ como unidad de muestreo, distribuido al azar, de forma paralela a la costa. Entre las expediciones de trabajo llevadas a cabo en el PNJR, en décadas pasadas, se escogieron los datos correspondientes a 2001, 2012 y 2017, por ser similares en cuanto a metodologías aplicadas. Además, dichas expediciones resultaron las de mayores números de sitios estudiados (Cuadro 1). 
Cuadro 1. Años, números de sitios y de transectos estudiados, número total de colonias y de colonias de $S$. siderea, Orbicella spp., A. agaricites y P. astreoides analizadas por años en los escarpes poco profundos del Parque Nacional Jardines de la Reina (PNJR)

Table 1. Years, number of sites and transects studied, total number of colonies and colonies of $S$. siderea, Orbicella spp., A. agaricites and P. astreoides analyzed by year in reef slopes of the Jardines de la Reina National Park (JRNP)

\begin{tabular}{|c|c|c|c|c|c|c|c|}
\hline Años & Sitios & \# de transectos & $\begin{array}{l}\text { \# Total de } \\
\text { colonias }\end{array}$ & $\begin{array}{l}\text { \# Colonias } \\
\text { S. siderea }\end{array}$ & $\begin{array}{c}\text { \# Colonias } \\
\text { Orbicella spp. }\end{array}$ & $\begin{array}{l}\text { \# Colonias } \\
\text { A. agaricites }\end{array}$ & $\begin{array}{l}\text { \# Colonias } \\
\text { P. astreoides }\end{array}$ \\
\hline 2001 & 14 & 88 & 1127 & 326 & 287 & 128 & 86 \\
\hline 2012 & 14 & 185 & 1883 & 588 & 271 & 262 & 213 \\
\hline 2017 & 24 & 240 & 3491 & 1032 & 466 & 498 & 381 \\
\hline Total & 52 & 513 & 6501 & 1946 & 1024 & 888 & 680 \\
\hline
\end{tabular}

Para conocer si $S$. siderea, Orbicella spp., $A$. agaricites y $P$. astreoides han experimentado variación temporal que pudiera interpretarse como un desplazamiento de grupos de especies dominantes en el PNJR, entre el 2001 y 2017, se estudiaron, estadísticamente, los indicadores ecológicos de abundancia relativa de colonias (\%) y densidad lineal promedio de colonias (colonias $10 \mathrm{~m}^{-1}$ ) (variables dependientes) para estas especies, en el 2001, 2012 y 2017 (variable independiente). Asimismo, se estudiaron los indicadores ecológicos de cobertura de coral vivo (\%) y el diámetro máximo promedio $(\mathrm{cm})$ (variables dependientes) de $S$. siderea, Orbicella spp., A. agaricites y $P$. astreoides, en el 2017, pues en los años anteriores estos valores se obtuvieron de forma general, sin ser especificados por especies. Para ello, se realizó la prueba no paramétrica de Kruskal Wallis, ya que los datos no cumplieron las premisas de normalidad y homogeneidad de varianza. En los análisis entre años y especies, en los cuales hubo diferencias significativas, se aplicó la prueba de Wilcoxon (nivel de significación de 0.05) para conocer entre qué años y especies existían aquellas. Los análisis estadísticos se realizaron con el programa Statistic 6.0 (StatSoft, Inc. 2004).

En este estudio, se consideró Orbicella spp. como el complejo que incluye a las especies $O$. annularis, Orbicella faveolata (Ellis \& Solander, 1786) y Orbicella franksi (Gregory, 1895). En el análisis integral, en el vocablo "corales", se incluyeron especies del género Millepora, orden Capitata, y del orden Scleractinia. Para la identificación de las especies, se tuvo presente lo expuesto por Zlatarski \& Martínez-Estalella (1980) y González-Ferrer (2004). 


\section{RESULTADOS}

En el PNJR, se contó un total de 6501 colonias de corales, durante los muestreos realizados en los años 2001, 2012 y 2017. Se identificó un total de 35 especies entre todos los corales: 16 en el 2001, 30 en el 2012 y 29 en el 2017. Por años, la abundancia relativa $(\%)$ presentó variaciones entre las distintas especies identificadas (Cuadro 2). Específicamente, S. siderea no tuvo diferencias significativas entre el 2001, 2012 y 2017 (29\%, 32\% y 29\%, respectivamente), con una abundancia general, entre los tres años, del $30 \%$ (Fig. 2A). Orbicella spp. mostró una disminución desde el 2001 al 2012 y 2017 (24\%, 14\% y 13\%, respectivamente), con diferencias significativas entre estos años (Fig. 2B) y una abundancia general del 16\%. A pesar que $S$. siderea indicó una abundancia mayor que Orbicella spp., en el 2001, esta diferencia no fue significativa (Kruskal-Wallis test: $\mathrm{H}(1, \mathrm{~N}=27)=$ $2.120604 P=0.1453)$.

Cuadro 2. Abundancia (\%) de las especies identificadas en los escarpes poco profundos del Parque Nacional Jardines de la Reina (PNJR), en el 2001, 2012 y 2017

Table 2. Abundance (\%) of the species identified in reef slopes of the Jardines de la Reina National Park (JRNP) in 2001, 2012 and 2017

\begin{tabular}{|c|c|c|c|}
\hline \multirow[t]{2}{*}{ Especies de corales } & \multicolumn{3}{|c|}{ Abundancia (\%) } \\
\hline & 2001 & 2012 & 2017 \\
\hline \multicolumn{4}{|l|}{ Phylum Cnidaria } \\
\hline \multicolumn{4}{|l|}{ Clase Anthozoa } \\
\hline \multicolumn{4}{|l|}{ Subclase Hexacorallia } \\
\hline \multicolumn{4}{|l|}{ Orden Scleractinea } \\
\hline \multicolumn{4}{|l|}{ Familia Acroporidae } \\
\hline Acropora cervicornis (Lamarck, 1816) & 0.44 & 0.10 & 0.37 \\
\hline Acropora palmata (Lamarck, 1816) & 0.17 & & \\
\hline \multicolumn{4}{|l|}{ Familia Agariciidae } \\
\hline Agaricia spp. & 0.17 & & \\
\hline Agaricia agaricites (Linnaeus, 1758) & 11.7 & 14.6 & 14.3 \\
\hline Agaricia humilis Verrill, 1902 & & 0.74 & 2.01 \\
\hline Agaricia lamarcki M. Edwards \& Haime, 1851 & & 0.32 & 0.86 \\
\hline Agaricia tenuifolia Dana, 1846 & & 0.05 & \\
\hline Agaricia fragilis Dana, 1848 & & 0.11 & 0.14 \\
\hline Leptoseris cucullata (Ellis \& Solander, 1786) & & 0.11 & 0.37 \\
\hline \multicolumn{4}{|l|}{ Familia Astrocoeniidae } \\
\hline Stephanocoenia intersepta (Lamarck, 1836) & 2.83 & 3.88 & 6.5 \\
\hline \multicolumn{4}{|l|}{ Familia Caryophylliidae } \\
\hline Eusmilia fastigiata (Pallas, 1766) & & 0.69 & 0.4 \\
\hline
\end{tabular}




\begin{tabular}{|c|c|c|c|}
\hline \multirow[t]{2}{*}{ Especies de corales } & \multicolumn{3}{|c|}{ Abundancia (\%) } \\
\hline & 2001 & 2012 & 2017 \\
\hline \multicolumn{4}{|l|}{ Familia Faviidae } \\
\hline Colpophyllia natans (Houttuyn, 1772) & 0.88 & 0.64 & 0.80 \\
\hline Diploria labyrinthiformis (Linnaeus, 1758) & 3.54 & 1.81 & 1.46 \\
\hline Favia fragum (Esper, 1795) & & & 0.06 \\
\hline Pseudodiploria clivosa (Ellis \& Solander, 1786) & 0.08 & & 0.29 \\
\hline Pseudodiploria strigosa (Dana, 1846) & 4.78 & 4.04 & 1.2 \\
\hline Montastraea cavernosa (Linnaeus, 1767) & 4.87 & 4.09 & 1.98 \\
\hline Orbicella spp. (complejo de especies) (Dana, 1846) & 24.4 & 14.1 & 13 \\
\hline \multicolumn{4}{|l|}{ Familia Meandrinidae } \\
\hline Dendrogyra cylindrus Ehrenberg, 1834 & & 0.05 & \\
\hline Dichocoenia stokesi M. Edwards \& Haime, 1848 & & 0.32 & 0.54 \\
\hline Meandrina meandrites (Linnaeus, 1758) & & 0.96 & 0.89 \\
\hline \multicolumn{4}{|l|}{ Familia Mussidae } \\
\hline Isophyllia rigida (Dana, 1846) & & 0.11 & 0.03 \\
\hline Mycetophyllia aliciae Wells, 1973 & & 0.05 & \\
\hline Mycetophyllia danaana M. Edwards \& Haime, 1849 & & 0.10 & 0.11 \\
\hline Mycetophyllia lamarckiana M. Edwards \& Haime, 1848 & & 0.26 & 0.29 \\
\hline Mussa angulosa (Pallas, 1766) & & 0.21 & \\
\hline \multicolumn{4}{|l|}{ Familia Pocilloporidae } \\
\hline Madracis decactis (Lyman, 1859) & & 0.26 & 0.27 \\
\hline Madracis auretenra Locke, Weil \& Coates, 2007 & & 0.31 & 0.14 \\
\hline \multicolumn{4}{|l|}{ Familia Poritidae } \\
\hline Porites astreoides Lamarck, 1816 & 7.8 & 10.3 & 12 \\
\hline Porites divaricata Lesueur, 1821 & & 0.95 & 0.52 \\
\hline Porites furcata Lamarck, 1816 & 1.51 & 0.26 & 0.40 \\
\hline Porites porites (Pallas, 1766) & 7.17 & 6.74 & 7.48 \\
\hline \multicolumn{4}{|l|}{ Familia Siderastreidae } \\
\hline Siderastrea siderea (Ellis \& Solander, 1786) & 29 & 32 & 29 \\
\hline Siderastrea radians (Pallas, 1766) & & & 1.23 \\
\hline \multicolumn{4}{|l|}{ Orden Capitata } \\
\hline \multicolumn{4}{|l|}{ Familia Milleporidae } \\
\hline Millepora alcicornis Linnaeus, 1758 & 0.62 & 1.96 & 2.84 \\
\hline Total de especies & 16 & 30 & 29 \\
\hline \multicolumn{4}{|c|}{ Especies más abundantes } \\
\hline & S. siderea & S. siderea & S. siderea \\
\hline & Orbicella spp. & A. agaricites & A. agaricites \\
\hline & A. agaricites & Orbicella spp. & Orbicella spp. \\
\hline & P. astreoides & P. astreoides & P. astreoides \\
\hline
\end{tabular}



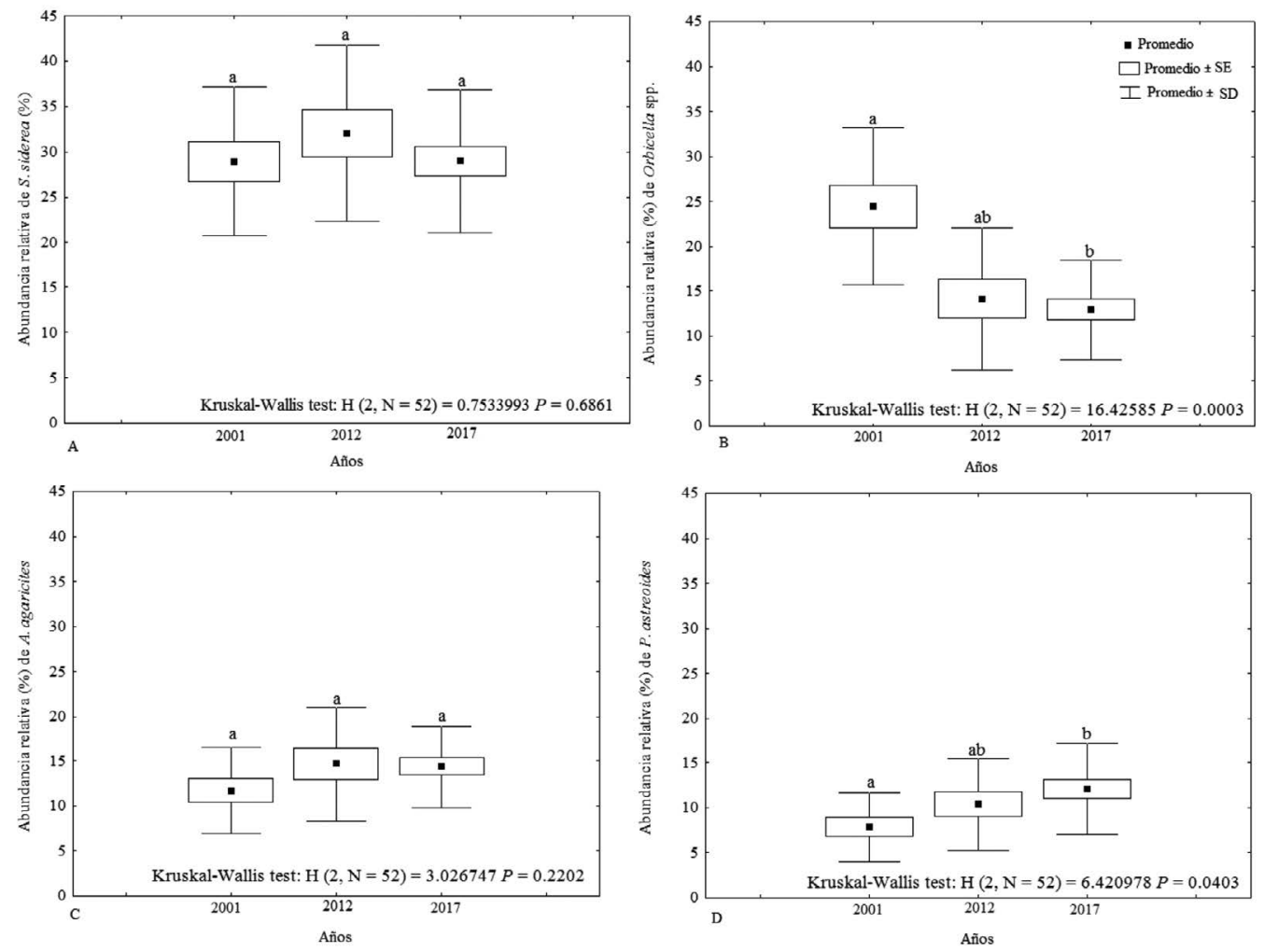

Fig. 2. Abundancia relativa (\%) de las especies $S$. siderea (A), Orbicella spp. (B), A. agaricites (C) y P. astreoides (D), en el 2001, 2012 y 2017, en el Parque Nacional Jardines de la Reina. Las letras, encima de las barras, significan los resultados de la prueba de Wilcoxon

Fig. 2. Relative abundance of the species $S$. siderea(A), Orbicella spp. (B), A. agaricites (C) and $P$. astreoides (D) in 2001, 2012 and 2017 in the Jardines de la Reina National Park. The letters above the bars indicate Wilcoxon test results

A. agaricites no tuvo diferencias significativas entre el 2001, 2012 y $2017(12 \%, 14.6 \%$ y $14 \%$, respectivamente), con una abundancia general de $14 \%$. P. astreoides evidenció un aumento de su abundancia entre el 2001, 2012 y $2017(8 \%, 10 \%$ y 12\%, respectivamente) (Fig. $2 \mathrm{C}$ y D), con una abundancia general, entre los tres años, del 10\%. Orbicella spp., A. agaricites y $P$. astreoides no mostraron diferencias significativas, entre ellas, para la abundancia relativa en el 2012 (Kruskal-Wallis test: $\mathrm{H}(2, \mathrm{~N}=42)=$ $3.322925 P=0.1899)$, ni en el 2017 (Kruskal-Wallis test: $\mathrm{H}(2, \mathrm{~N}=72)=$ $2.487650 P=0.2883)$. 
S. siderea mantuvo una densidad lineal promedio sin diferencias significativas entre el 2001 (3.7 colonias 10 $\left.\mathrm{m}^{-1}\right), 2012\left(3.2\right.$ colonias $\left.10 \mathrm{~m}^{-1}\right)$ y 2017 (4.3 colonias $10 \mathrm{~m}^{-1}$ ) (Fig. $3 \mathrm{~A}$ ), con un valor total, entre los tres años, de 3.8 colonias $10 \mathrm{~m}^{-1}$. La densidad de Orbicella spp. fue menor en el 2012 (1.4 colonias $\left.10 \mathrm{~m}^{-1}\right)$ y 2017 (1.9
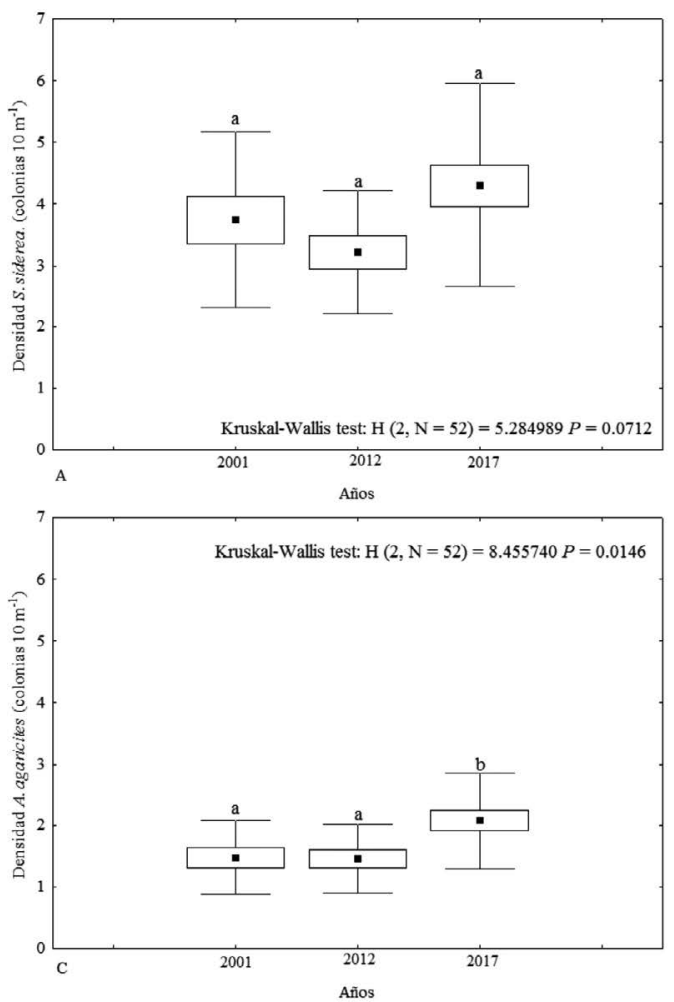

colonias $\left.10 \mathrm{~m}^{-1}\right)$, en comparación con el 2001 (3.2 colonias $\left.10 \mathrm{~m}^{-1}\right)$ (Fig. 3 B), con un valor total de 2.2 colonias $10 \mathrm{~m}^{-1}$. A pesar de que $S$. siderea dejó ver una densidad mayor que Orbicella spp., en el 2001, esta diferencia no fue significativa (Kruskal-Wallis test: $\mathrm{H}$ $(1, \mathrm{~N}=28)=1.978554 P=0.1595)$.
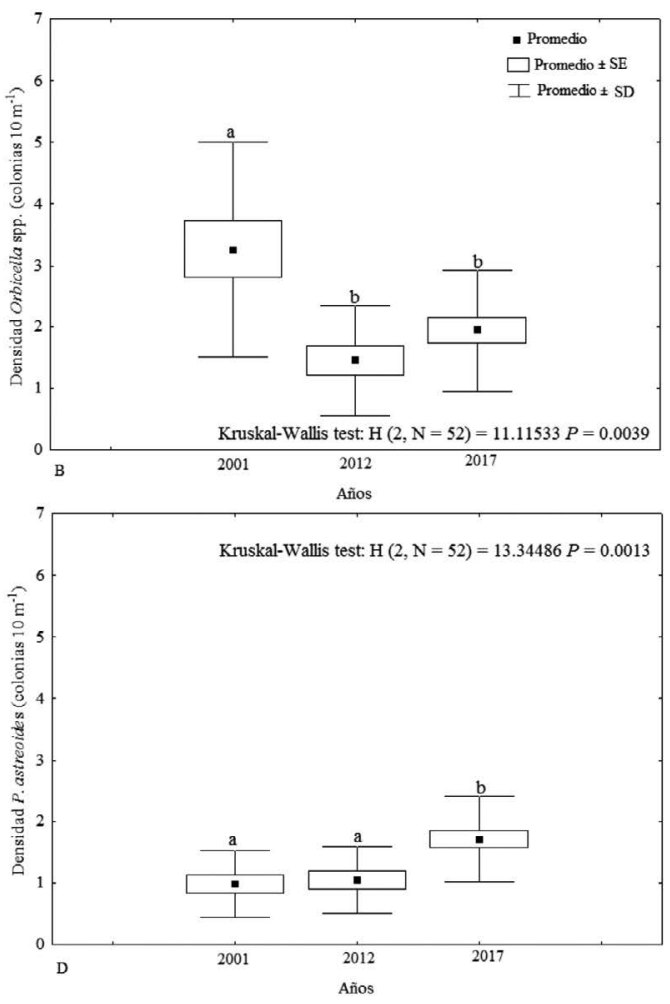

Fig. 3. Densidad (colonias $10 \mathrm{~m}^{-1}$ ) de las especies $S$. siderea (A), Orbicella spp. (B), A. agaricites (C) y P. astreoides (D), en el 2001, 2012 y 2017, en el Parque Nacional Jardines de la Reina. Las letras, encima de las barras, significan los resultados de la prueba de Wilcoxon

Fig. 3. Density (colonies $10 \mathrm{~m}^{-1}$ ) of the species $S$. siderea (A), Orbicella spp. (B), A. agaricites (C), and P. astreoides (D) in 2001, 2012 and 2017 in the Jardines de la Reina National Park. The letters above the bars indicate Wilcoxon test results 
A. agaricites conservó igual densidad entre el 2001 y 2012 (1.4 colonias $10 \mathrm{~m}^{-1}$ ) y la aumentó en el 2017 (2 colonias $10 \mathrm{~m}^{-1}$ ), con diferencias significativas respecto a los años anteriores, con una densidad general de 1.7 colonias $10 \mathrm{~m}^{-1}$. P. astreoides manifestó una densidad de 1 colonia $10 \mathrm{~m}^{-1}$, en el 2001 y 2012, mientras que en el 2017 incrementó a 1.7 colonias $10 \mathrm{~m}^{-1}$ y su densidad general fue de 1.3 colonias $10 \mathrm{~m}^{-1}$, entre los tres años (Fig. 3 C y D). Orbicella spp., A. agaricites y $P$. astreoides no expresaron diferencias significativas, entre ellas, para la densidad lineal promedio en el 2012 (Kruskal-Wallis test: $\mathrm{H}(2, \mathrm{~N}=42)=$ $3.267151 P=0.1952)$ ni en el 2017
(Kruskal-Wallis test: $\mathrm{H}(2, \mathrm{~N}=72)=$ $1.952243 P=0.3768$ ).

En el 2017, Orbicella spp. tuvo una cobertura, significativamente, mayor (19\%) al resto de las especies, seguida de $S$. siderea (16\%). A. agaricites (12\%) y P. astreoides (11\%) no dataron diferencias significativas entre ellas para este indicador ecológico (Fig. 4A). El diámetro máximo promedio de las colonias fue, significativamente, mayor en Orbicella spp. $(30 \mathrm{~cm})$, respecto al resto de las especies: $S$. siderea $16 \mathrm{~cm}$, A. agaricites $14 \mathrm{~cm}$ y $P$. astreoides $18 \mathrm{~cm}$ - no expusieron diferencias significativas entre sí (Fig. 4B)—.
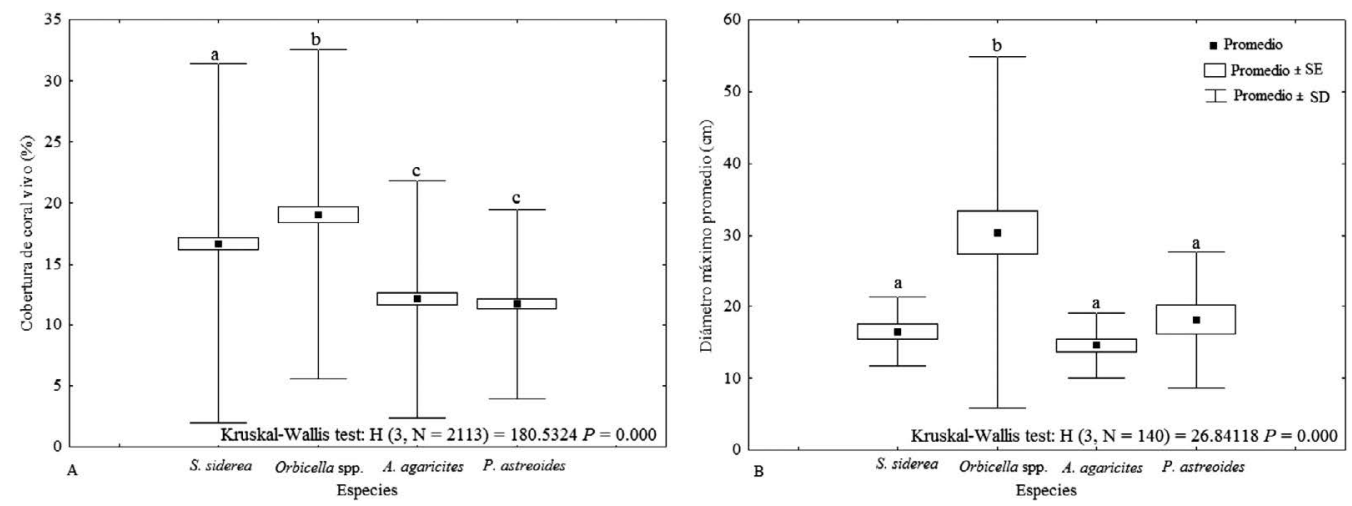

Fig. 4. Cobertura (\%) (A) y diámetro máximo promedio (cm) (B) de las especies S. siderea, Orbicella spp., A. agaricites y P. astreoides, en el Parque Nacional Jardines de la Reina, en el 2017. Las letras, encima de las barras, significan los resultados de la prueba de Wilcoxon

Fig. 4. Cover (\%) (A) and maximum average diameter (cm) (B) of the species $S$. siderea, Orbicella spp., A. agaricites, and P. astreoides in Jardines de la Reina National Park in 2017. The letters above the bars indicate Wilcoxon test results 


\section{DISCUSIÓN}

El número de especies identificadas en este estudio estuvo por debajo del reportado por González-Díaz et al. (2018) - 37 especies - y Hernández-Fernández et al. (2019a) —40 especies - para el PNJR.

La composición de las especies dominantes de corales pétreos, en los escarpes poco profundos, coincide, en parte, con la de los arrecifes del Parque Nacional Guanahacabibes, donde predominan $S$. siderea, A. agaricites y $P$. astreoides, además de la especie $O$. faveolata (Perera-Valderrama et al. 2013; 2017). Para otros arrecifes de Cuba, según González-Díaz et al. (2018), también imperan S. siderea, A. agaricites y $P$. astreoides, así como Stephanocoenia intersepta (Lamarck, 1816). Estos autores consideraron que Orbicella spp., junto con Acropora cervicornis (Lamarck, 1816), son especies poco común en los arrecifes cubanos. Específicamente, en este estudio, A. cervicornis mostró valores bajos de abundancia, acentuados en el 2012, aunque hubo especies que tuvieron una abundancia menor, como Pseudodiploria clivosa (Ellis \& Solander, 1786), Isophyllia rigida (Dana, 1846) y Dendrogyra cylindrus Ehrenberg, 1834, entre otras; esta última especie es la de menor abundancia en el PNJR. Asimismo, Caballero et al. (2019), en un trabajo sobre las condiciones ecológicas de los arrecifes coralinos en el archipiélago cubano, identificaron a $S$. siderea, A. agaricites, Orbicella spp. y $P$. astreoides, en este orden, como las especies más abundantes para los hábitats de escarpes poco profundos, lo que coincide con los resultados obtenidos en esta investigación.

Según Rey-Villiers et al. (2016), en el PNJR, el patrón del predominio de las especies, en los escarpes poco profundos, estaba cambiando desde las típicas formadoras de arrecifes, como $O$. annularis hacia especies oportunistas como $S$. siderea, $P$. astreoides y $A$. agaricites. Sin embargo, la abundancia y la densidad de $S$. siderea no mostraron diferencias significativas con la de Orbicella spp. en el 2001, por lo que $S$. siderea ha mantenido la dominancia en estos arrecifes, sin que por ello haya desplazado a Orbicella spp. Lo anterior no coincide con lo planteado por Alcolado et al. (2009), para el golfo de Batabanó, y Caballero et al. (2019), para arrecifes cubanos; ellos consideraron que $S$. siderea ha desplazado a Orbicella spp. En el PNJR no ha aumentado la abundancia y densidad de S. siderea, han disminuido estos indicadores ecológicos en Orbicella spp. No obstante, en tiempos pasados (antes de 2001), puede ser que dominara Orbicella spp. por encima de $S$. siderea, pero esto, por ahora, no ha sido demostrado. Tal hipótesis está soportada por lo que exponen Toth et al. 
(2019), quienes, en un estudio realizado en la Florida, llegaron a la conclusión de que hace miles de años (en el Holoceno), Orbicella spp. y otros corales masivos representaban casi el $90 \%$ de los arrecifes, mientras que $S$. siderea y $P$. astreoides eran especies raras, por lo que sugieren que las modificaciones recientes, en conjuntos de especies, no tienen precedentes en escalas de tiempo milenarias.

$S$. siderea es un coral masivo, constructor de arrecifes, tiene larga vida, es tolerante a los cambios térmicos y también común en el Caribe (De Bakker et al. 2016; Benson et al. 2018). Resiste a los cambios de salinidad (Muthiga \& Szmant, 1987) y de temperatura, lo que permite que generalmente sobreviva a los eventos de blanqueamiento (Banks \& Foster, 2017). Todas estas características pueden ser las razones por las cuales ha mantenido su dominancia en el PNJR. La abundancia de $S$. siderea en el PNJR fue similar a la determinada en Puerto Morelos, en el norte del Caribe Mexicano, (35.5\%) en julio de 2018, aunque esta declinó a $14.9 \%$, en solo ocho meses, ante la presencia de la enfermedad de pérdida del tejido del coral duro (SCTLD) (por sus siglas en inglés) (Estrada-Saldíva et al. 2020).

Los cambios en la abundancia de los corales involucran a especies que han sufrido enfermedades, hecho que altera su representación regional (Darling et al. 2012). En el PNJR, no hay evidencias de afectaciones por SCTLD $\mathrm{u}$ otras enfermedades, sobre la comunidad de corales pétreos, que pudieran justificar la disminución de la abundancia relativa y la densidad lineal de Orbicella spp. desde el 2001 hasta el 2012. Por ejemplo, uno de los mayores eventos de blanqueamiento ocurridos en el Caribe fue el del 2005 (Steiner \& Kerr, 2008), que tuvo una incidencia pobre en la comunidad de corales pétreos del parque y con el cual hubo especies que demostraron evidentes signos de recuperación, como Orbicella spp. (en aquel momento nombrado el género Montastraea) cuyas colonias, en cuatro meses, manifestaron una recuperación total (Hernández-Fernández et al. 2011). Precisamente, las afectaciones por blanqueamiento son una de las razones por las que las poblaciones de Orbicella spp. están declinando en el Caribe (Hayes, 2019; Toth et al. 2019; Yranzo, 2020), pues sus colonias no muestran la misma capacidad de respuesta, en todas las regiones (Kennedy et al. 2019). Sin embargo, A. agaricites mostró una recuperación parcial al blanqueamiento en el PNJR, comprobando ser una de las especies más sensibles a este (Hernández-Fernández et al. 2011). $A$. agaricites, al igual que $S$. siderea, no tuvo diferencias significativas, entre el 2001, 2012 y 2017, en cuanto a la abundancia relativa, no así para la densidad, la cual aumentó en el 2017, en comparación con el 2001 y 2012. 
Más recientemente, en una investigación realizada en el PNJR sobre la variabilidad a pequeña escala de la cobertura y diversidad de las especies bentónicas dominantes (Hernández-Fernández et al. 2019b), no se observó la presencia de enfermedades. Las variaciones en los arrecifes, cuando no pueden ser explicadas por variables ambientales, tal vez tengan respuestas en disturbios antrópicos que inciden a nivel local (Ferrigno et al. 2016). Pero los arrecifes del PNJR no están afectados por agentes estresantes (González-Díaz et al. 2018), a pesar de que en ellos se desarrolla el buceo recreativo (Hernández-Fernández et al. 2016). Por ello, es necesaria la realización de un estudio integral, en el cual se analice la incidencia de los estresores globales, fundamentalmente, de los huracanes y del incremento de temperatura de la superficie del mar, sobre los arrecifes coralinos del PNJR, y la posible resiliencia ante estos, más aún si estos resultados persisten en el tiempo. El declive de Orbicella spp. también puede estar asociado a episodios de enfermedades (Hayes, 2019; Toth et al. 2019; Yranzo, 2020), causadas por estresores globales, estudio que por el momento no se ha realizado en el PNJR. Precisamente, Hernández-Fernández et al. (2019b) consideraron que la condición actual de los arrecifes del PNJR (tanto dominancia de algas como baja cobertura y diversidad de corales) hace pensar en un cambio de fase continuado, que se vincula, primordialmente, a los efectos del estrés global sobre el arrecife.

El aumento de la abundancia relativa de $P$. astreoides corresponde con los resultados obtenidos en otras islas del Caribe, los que han evidenciado un aumento relativo en la abundancia de esta especie, en zonas menos profundas del arrecife (Green et al. 2008). En un trabajo realizado por Edmunds (2010), en arrecifes de esta región, se pudo constatar que la población de $P$. astreoides se acrecentó gradualmente entre 1999 y el 2007, e incluso pronosticó que en los próximos 100 años su población aumentará en abundancia. También, específicamente, en los arrecifes de las islas de Curacao y Bonaire hubo un incremento en las poblaciones de $P$. astreoides y otras especies oportunistas, desde 1973 hasta el 2014, lo que ha ocasionado la declinación en estos arrecifes (De Bakker et al. 2016). Sin embargo, otros estudios como los realizados en Granada mostraron un decrecimiento de la densidad media de colonias de esta especie, entre el 2014 y 2017 (Eagleson et al. 2021). Justamente, $P$. astreoides es una de las especies consideradas como "weedy", lo que contribuye al aumento de su abundancia (Holstein et al. 2016) y ha sido uno de los componentes típicos de los arrecifes coralinos del Caribe durante milenios (Pandolfi \& Jackson 2006).

A pesar de la degradación de Orbicella spp., ante los indicadores 
ecológicos de abundancia y densidad, en el 2017, esta especie reveló mayor porcentaje de cobertura que las restantes especies analizadas, lo que está en correspondencia con el diámetro máximo promedio imperante en ella. En una investigación elaborada en Punta Maroma, península de Yucatán, Medina-Valmaseda et al. (2020) determinaron un porcentaje de cobertura para Orbicella spp. (12.6\%), menor al obtenido para el PNJR en el 2017. Estos autores detectaron una disminución en el diámetro máximo promedio de las colonias de Orbicella spp. entre 1985 y 2019; obtuvieron un valor, en este último año, de aproximadamente $18.6 \mathrm{~cm}$, por debajo del alcanzado en el PNJR, en el 2017. Por su parte, el diámetro promedio de $A$. agaricites, obtenido en el PNJR, se puede considerar pequeño, teniendo en cuenta que las colonias adultas podrían alcanzar hasta $60 \mathrm{~cm}$ (Helmuth \& Sebens, 1993). Esta especie es considerada también como "weedy" y ha disminuido su cobertura en el Caribe, desde 1973 hasta el 2014 , de un $9.9 \%$ a $5.2 \%$ (De Bakker et al. 2016), valores que se encuentran por debajo del obtenido para el PNJR en el 2017. Precisamente, los cambios en las poblaciones de corales es posible que se caractericen por una transición a especies "weedy" (Darling et al. 2012) o estar asociados al incremento de las tallas de las colonias (Hughes \& Jackson, 1985). Igualmente, pueden estar asociados a la disminución de las tallas de las colonias, lo cual estaría en dependencia de los impactos antrópicos o naturales imperantes y de las condiciones ambientales prevalecientes en cada arrecife. En el PNJR, no hay estudios que demuestren que hayan existido modificaciones en la población de corales, relacionadas a las tallas de los corales.

En el PNJR, específicamente, en el hábitat de los escarpes poco profundos, persiste la dominancia de S. siderea en el tiempo (2001-2017), por lo que no se debe decir que esta especie ha sustituido a Orbicella spp. Más que un incremento de especies "weedy", donde no se incluye a $S$. siderea y sí a $A$. agaricites y $P$. astreoides (De Bakker et al. 2016), lo que ha ocurrido, particularmente, desde el 2001 hasta el 2012, ha sido un decrecimiento en la abundancia y densidad de Orbicella spp., sin causas aún precisadas, período que coincide con el expuesto por Green et al. (2008), quienes plantean que la estructura de la comunidad de los arrecifes coralinos, en el Caribe, se está transformando sobre una escala de diez años. Esta mengua de Orbicella spp. no continuó hasta el 2017, pero se requeriría cinco años más, a partir de 2017, para evaluar nuevamente estos indicadores ecológicos y conocer si sus resultados persisten o no en el tiempo. Ello, junto con el aumento significativo de la densidad de $A$. agaricites y el incremento significativo tanto de la 
abundancia como de la densidad de P. astreoides, desde el 2012 al 2017 (con valores, en el 2017, que no se diferencian, significativamente, con los obtenidos para Orbicella spp.), es una evidencia de una variación temporal en la comunidad de corales pétreos en el PNJR. Dicha modificación puede interpretarse como un desplazamiento, en el grupo de especies dominantes, de Orbicella spp. hacia A. agaricites y $P$. astreoides, lo cual coincide con lo que está ocurriendo en otros arrecifes del Caribe (Jackson et al. 2014; De Bakker et al. 2016).

Aunque los cambios en el ecosistema no son fáciles de predecir (porque pueden suceder en poco tiempo) y la disminución de las poblaciones de corales podría tener dificultad para revertirse (Mumby \& Steneck, 2011), los resultados de este trabajo constituyen una alerta para los tomadores de decisiones del área protegida, a los cuales se les sugiere continuar los estudios sobre las poblaciones de Orbicella spp., A. agaricites y $P$. astreoides, y así prever una degradación del arrecife, en caso que los resultados mostrados aquí, persistan.

\section{AGRADECIMIENTOS}

Se agradece a todos aquellos que participaron en la expedición CUBAGRRA II, efectuada en agosto del 2001. También, a los que formaron parte de las expediciones realizadas en abril del 2012 y septiembre-octubre del 2017, pertenecientes al Centro de Investigaciones de Ecosistemas Costeros (CIEC), al Centro de Investigaciones Marinas (CIM) y al Instituto de Oceanología de Cuba. Igualmente, gracias a todo el personal de la Sucursal Marlin Jardines de la Reina y, en específico, al de la embarcación "Oceans For Youth". Un agradecimiento especial a Roberto González-De Zayas, Fabián Pina Amargós, Claudia Bustamante López, Roy Phillips Pérez, Evelio A. Alemán Martín, Noel López y Eduardo del Sol Cruz. Asimismo, se agradece a la ONG Idea Wild, por facilitar medios para la realización del trabajo, y a los revisores anónimos, por sus atinadas e importantes sugerencias.

\section{REFERENCIAS}

AGRRA. (2000). The AGRRA Rapid Reef Assessment Protocol. Atlantic and Gulf Rapid Reef Assessment Methodology. https://www.agrra.org/

Alcolado, P. M., Hernández-Muñoz, D., Caballero, H., Busutill, L., Perera, S. \& Hidalgo, G. (2009). Efectos de un inusual período de alta frecuencia de huracanes sobre el bentos de arrecifes coralinos. Rev. Mar. Cost., 1, 73-93. https://doi. org/10.15359/revmar.1.4

Banks, S. \& Foster, K. (2017). Baseline Levels of Siderastrea siderea Bleaching under Normal Environmental Conditions in Little Cayman. Open J. Mar. Sci., 7, 142-154. http://dx.doi.org/10.4236/ ojms.2017.71011

Baumann, J. H., Townsend, J. E., Courtney, T. A., Aichelman, H. E., Davies, S. W., 
Lima, F. P. \& Castillo, K. D. (2016). Temperature Regimes Impact Coral Assemblages along Environmental Gradients on Lagoonal Reefs in Belize. PloS ONE, 11, 9, e0162098. https://doi. org/10.1371/journal.pone.0162098

Benson, E. B., Rippe, P. J., Bove1, B. C. \& Castillo, D. K. (2018). Apparent timing of density banding in the Caribbean coral Siderastrea siderea suggests complex role of key physiological variables. Coral Reefs, 38, 165-176. https:// doi.org/10.1007/s00338-018-01753-w

Bruno, J. F., Coté, I. M. \& Toth, L. T. (2019). Climate change, coral loss, and the curious case of the parrotfish paradigm: why don't marine protected areas improve reef resilience? Annu. Rev. Mar. Sci., 11, 307-334. https://doi.org/10.1146/ annurev-marine-010318-095300

Caballero, H., Armenteros, M., Perera Valderrama, S., Rey Villiers, N., Cobián Rojas, D., Campos Verdecia, K. \& Alcolado Menéndez, P. M. (2019). Ecological condition of coral reef assemblages in the Cuban Archipelago. Mar. Biol. Res., 15(1), 61-73. https://doi.org/10.1 080/17451000.2019.1577557

Caballero, H., Alcolado, P. M., González, P., Perera, S. \& Hernández-Fernández, L. (2013). Protocolo para el monitoreo de bentos en arrecifes coralinos. Versión ajustada a partir del método de campo AGRRA 2000. Cuba: Centro Nacional de Áreas Protegidas.

Darling, E. S., Álvarez-Filip, L., Oliver, T. A., McClanahan, T. R. \& Coté, I. M. (2012). Evaluating life-history strategies of reef corals from species traits. Ecol. Lett., 15(12), 1378-1386. https://doi. org/10.1111/j.1461-0248.2012.01861.x

De Bakker, D. M., Meesters, E. H., Bak, R. P. M., Nieuwland, G. \& Van Duyl, F. C. (2016). Corrigendum: Long term shift in coral communities on shallow to deep reef slopes of Curacao and Bonaire: are there any winners? Front. Mar. Sci., 3, 247. https://doi.org/10.3389/ fmars.2017.00171

Eagleson, R. G., Lumsden, J. S., Álvarez-Filip, L., Herbinger, C. M. \& Horricks, R. A. (2021). Coverage Increases of Porites astreoides in Grenada Determined by Shifts in Size-Frequency Distribution. Diversity, 13(288), 1-15. https:// doi.org/10.3390/d13070288

Edmunds, P. J. (2015). A quarter-century demographic analysis of the Caribbean coral, Orbicella annularis, and projections of population size over the next century. Limnol. Oceanogr., 60(3), 840-855. https://doi.org/10.1002/lno.10075

Edmunds, P. J. (2010). Population biology of Porites astreoides and Diploria strigo$s a$ on a shallow Caribbean reef. Mar. Ecol. Prog. Ser., 418, 87-104. https:// doi.org/10.3354/meps08823

Estrada-Saldívar, E., Molina-Hernández, A., Pérez-Cervantes, E., Medellín-Maldonado, F., González-Barrios, J. F. \& Álvarez-Filip, L. (2020). Reef-scale impacts of the stony coral tissue loss disease outbreak. Coral Reefs, 39, 861-866. https://doi.org/10.1007/ s00338-020-01949-z

Ferrer-Rodríguez, V. M., González-Díaz, P., Hernández-Fernández, L., Siciliano, D., Bretos, F., Appril, A., ... \& Santoro, A. (2016). Salud de las comunidades de corales en arrecifes de Jardines de la Reina - Golfo de Ana María, región surcentral de Cuba. Rev. Inv. Mar., 36(1), 34-56.

Ferrigno, F., Bianchi, C. N., Lasagna, R., Morri, C., Russo, G. F. \& Sandulli, R. (2016). Corals in high diversity reefs resist human impact. Ecol. Indic., 70, 106-113. https://doi.org/10.1016/j. ecolind.2016.05.050 
Gil-Agudelo, D. L., Cintra-Buenrostro, C. E., Brenner, J., González-Díaz, P., Kiene, W., Lustic, C. \& Pérez-España, H. (2020). Coral Reefs in the Gulf of Mexico Large Marine Ecosystem: Conservation Status, Challenges, and Opportunities. Front. Mar. Sci., 6(807), 1-20. https://doi. org/10.3389/fmars.2019.00807

González-Díaz, P., González-Sansón, G., Aguilar-Betancourt, C., Álvarez-Fernández, S., Perera-Pérez, O., Hernández-Fernández, L., \& ... de la Guardia-Llanso, E. (2018). Status of Cuban coral reefs. Bull. Mar. Sci., 94, 229-247. https://doi.org/10.5343/bms.2017.1035

González-Ferrer, S. (2004). Catálogo de los corales hermatípicos de aguas cubanas. En S. González-Ferrer (Ed.), Corales pétreos. Jardines sumergidos de Cuba (pp. 79-189). España: Editorial Academia.

Goodbody-Gringley, G. \& de Putron, S. J. (2016). Brooding Corals: Planulation Patterns, Larval Behavior, and Recruitment Dynamics in the Face of Environmental Change. In S. Goffredo \& $\mathrm{Z}$. Dubinsky (Eds.), The Cnidaria, Past, Present and Future (pp. 279-289). Switzerland: Springer.

Green, H. D., Edmunds, J. P. \& Carpenter, C. R. (2008). Increasing relative abundance of Porites astreoides on Caribbean reefs mediated by an overall decline in coral cover. Mar. Ecol. Prog. Ser., 359, 1-10. https://doi.org/10.3354/meps07454

Hayes, N. K. (2019). A characterization of a Southeast Florida stony coral assemblage after a diseaseevent. (Unpublished master's thesis). Nova Southeastern University.

Helmuth, B. \& Sebens, K. (1993). The influence of colony morphology and orientation to flow on particle capture by the scleractinian coral Agaricia agaricites (Linnaeus). J. Exp. Mar. Biol. Ecol., 165(2), 251-278. https://doi. org/10.1016/0022-0981(93)90109-2
Hernández-Fernández, L., Bustamante López, C., Dulce Sotolongo, L. B., Pina Amargós, F. \& Figueredo, T. (2019a). Influencia del gradiente de protección sobre el estado de las comunidades de corales y algas coralinas costrosas en el Parque Nacional Jardines de la Reina, Cuba. Rev. Invest. Mar., 38(1), 83-99.

Hernández-Fernández, L., González de Zayas, R., Weber, L., Apprill, A. \& Armenteros, M. (2019b). Small-Scale Variability Dominates Benthic Coverage and Diversity Across the Jardines de La Reina, Cuba Coral Reef System. Front. Mar. Sci., 6, 747, 1-12. https://doi. org/10.3389/fmars.2019.00747

Hernández-Fernández, L., Olivera, Y. M., Figueredo-Martín, T., Gómez Fernández, R., Brizuela-Pardo, L. \& Pina-Amargós, F. (2016). Incidencia del buceo autónomo y capacidad de carga en sitios de buceo del Parque Nacional Jardines de la Reina, Cuba. Rev. Mar. Cost., 8(2), 9-27. https://doi.org/10.15359/ revmar.8-2.1

Hernández-Fernández, L., Guimarais Bermejo, M., Arias Barreto, R. \& Clero Alonso, L. (2011). Composición de las comunidades de octocorales y corales pétreos y la incidencia del blanqueamiento del 2005 en Jardines de la Reina, Cuba. Rev. Mar. Cost., 3, 77-90. https://doi.org/10.15359/revmar.3.6

Hoegh-Guldberg, O., Mumby, P. J., Hooten, A. J., Steneck, R. S., Greenfield, P., Gomez, E., ... \& Hatziolos, E. (2007). Coral reefs under rapid climate change and ocean acidification. Science, 318(5857), 1737-1742. https://doi. org/10.1126/science. 1152509

Hoegh-Guldberg, O., Pendleton, L. \& Kaup, A. (2019). People and the changing nature of coral reefs. Reg. Stud. Mar. Sci., 30, 1-20. https://doi.org/10.1016/j. rsma.2019.100699 
Holstein, D. M., Smith, T. B. \& Paris, C. B. (2016). Depth-independent reproduction in the reef coral Porites astreoides from shallow to mesophotic zones. PLoS One, 11, e0146068. https://doi. org/10.1371/journal.pone. 0146068

Hughes, T. P. \& Jackson, B. C. J. (1985). Population dynamics and life histories of foliaceous corals. Ecol. Monogr., 55(2), 141-166. https://doi. org/10.2307/1942555

Hughes, T. P., Graham, A J. N., Jackson, B. C. J., Mumby, J. P. \& Steneck, S. R. (2010). Rising to the challenge of sustaining coral reef resilience. Trends. Ecol. Evo., 25(11), 633-642. https:// doi.org/10.1016/j.tree.2010.07.011

Jackson, J., Donovan, M., Cramer, K. \& Lam, V. (2014). Status and Trends of Caribbean Coral Reefs: 1970-2012. Switzerland: Global Coral Reef Monitoring Network.

Kennedy, V. E., Tonk, L., Foster, L. N., Mumby, J. P. \& Stevens, R. J. (2019). Temporal stability of Orbicella annularis symbioses: a case study in The Bahamas. Bull. Mar. Sci., 95(2), 289-304. https://doi.org/10.5343/bms.2018.0064

Medina-Valmaseda, A. E., Rodríguez-Martínez, R. E., Álvarez-Filip, L., Jordan-Dahlgren, E. \& Blanchon, P. (2020). The role of geomorphic zonation in long-term changes in coral-community structure on a Caribbean fringing reef. PeerJ., 8, e10103. https://doi.org/10.7717/peerj.10103

Mumby, J. P. \& Steneck, S. R. (2011). The Resilience of Coral Reefs and Its Implications for Reef Management. In Z. Dubinsky \& N. Stambler (Eds.), Coral Reefs: An Ecosystem in Transition (pp. 509-519). EE. UU: Springer.

Muthiga, N. A. \& Szmant, A. M. (1987). The effects of salinity stress on the rates of aerobic respiration and photosynthesis in the hermatypic coral Siderastrea siderea. Biol. Bull., 173(3), 539-551. https://doi.org/10.2307/1541699

Obura, D. O. \& Grimsdith, G. (2009). Resilience Assessment of coral reefs-Assessment protocol for coral reefs, focusing on coral bleaching and thermal stress. Switzerland: IUCN working group on Climate Change and Coral Reefs.

Pandolfi, J. M. \& Jackson, J. B. C. (2006). Ecological persistence interrupted in Caribbean coral reefs. Ecol Lett., 9(7), 818-826. https://doi. org/10.1111/j.1461-0248.2006.00933.x

Perera Valderrama, S., Alcolado, P. M., Caballero-Aragón, H., de la Guardia Llansó, E. \& Cobián- Rojas, D. (2013). Condición de los arrecifes coralinos del Parque Nacional Guanahacabibes, Cuba. Rev. Mar. Cost., 5, 69-86. https://doi. org/10.15359/revmar.5.5

Perera Valderrama, S., Hernández-Arana, H., Ruiz-Zárate, M. A., Alcolado, P. M., Caballero-Aragón, H., González-Cano, J., .. \& \& de la Guardia Llansó, E. (2017). Temporal dynamic of reef benthic communities in two marine protected areas in the Caribbean. J. Sea Res., 128, 15-24. https://doi.org/10.1016/j. seares.2017.07.007

Perera-Valderrama, S., Ávila, A. H., Méndez, J. G., Martínez, O. M., Rojas, D. C., Ferro Azcona, H. \& Rodríguez Farrat, L. F. (2018). Marine protected areas in Cuba. Bull. Mar. Sci., 94(2), 423-442. https://doi.org/10.5343/bms.2016.1129

Pina-Amargós, F., Hernández-Fernández, L., Clero, L. \& González-Sansón, G. (2008). Características de los hábitats coralinos en Jardines de la Reina, Cuba. Rev. Invest. Mar., 29, 225-237.

Rey-Villiers, N., Alcolado-Prieta, P., Busutil, L., Caballero, H., Perera-Pérez, O., Hernández-Fernández, L., ... \& Alcolado, M. P. (2016). Condición de los 
arrecifes coralinos del golfo de Cazones y el archipiélago Jardines de la Reina, Cuba: 2001-2012. En N. Rey-Villiers (Ed.), Línea base ambiental para el estudio del cambio climático en el golfo de Cazones y el archipiélago Jardines de la Reina, Cuba (pp. 93-146). Cuba: Instituto de Oceanología, CITMA.

StatSoft, Inc. (2004). STATISTICA for Windows [Computer program manual]. EE.UU.: StatSoft, Inc.

Steiner, S. C. C. \& Kerr, J. M. (2008). Stony corals in Dominica during the 2005 bleaching episode and one year later. Rev. Biol. Trop., 56 (Supple. 1), 139-148. https://doi.org/10.15517/rbt. v56i0.5583

Toth, T. L., Stathakopoulos, A., Kuffner B. I., Ruzicka, R. R., Colella, A. M. \& Shinn,
A. E. (2019). The unprecedented loss of Florida's reef-building corals and the emergence of a novel coral-reef assemblage. Ecol., 100(9), e02781. https://doi. org/10.1002/ecy.2781

van Woesik, R. \& Randall, C. J. (2017). Coral disease hotspots in the Caribbean. Ecosphere., 8(5), https://doi. org/10.1002/ecs 2.1814

Yranzo, A. (2020). Coral pilar estrella y coral estrella montañoso Orbicella annularis y Orbicella faveolata, Venezuela. Venezuela: Instituto de Zoología y Ecología Tropical.

Zlatarski, V. N. \& Martínez-Estalella, N. (1980). Escleractíneos de Cuba con datos sobre los organismos asociados (en ruso). Bulgaria: Editorial Academia de Bulgaria. 